\title{
Civilisations
}

Revue internationale d'anthropologie et de sciences

humaines

$66 \mid 2017$

L'alcool rituel et les ethnographes

\section{Une « ivresse rituelle »? Ethnographie croisée de rites alcoolisés, entre chamanisme asiatique et judaïsme européen (Népal - France)}

\section{Lionel Obadia}

\section{(2) OpenEdition \\ 1 Journals}

\section{Édition électronique}

URL : http://journals.openedition.org/civilisations/4380

DOI : 10.4000/civilisations.4380

ISSN : 2032-0442

Éditeur

Institut de sociologie de l'Université Libre de Bruxelles

\section{Édition imprimée}

Date de publication : 31 août 2017

Pagination : 91-104

ISBN : 978-2-9602017-1-0

ISSN : 0009-8140

\section{Référence électronique}

Lionel Obadia, «Une « ivresse rituelle »? Ethnographie croisée de rites alcoolisés, entre chamanisme asiatique et judaïsme européen (Népal - France) », Civilisations [En ligne], 66 | 2017, mis en ligne le 31 août 2020, consulté le 25 février 2021. URL : http://journals.openedition.org/civilisations/4380 ; DOI https://doi.org/10.4000/civilisations.4380 


\title{
Une « ivresse rituelle »? \\ Ethnographie croisée de rites alcoolisés, entre chamanisme asiatique et judaïsme européen (Népal - France)
}

\author{
Lionel OBADIA
}

Résumé : A partir de la présentation ethnographique de deux rituels très différents dans leur forme et dans leurs contextes géographiques, culturels et religieux - le Pessah juif en Europe et le guru-puja chamanique au Népal-, cet article s'attache à montrer les contrastes morphologiques, fonctionnels et symboliques non pas des rituels eux-mêmes, mais des modalités de l'alcoolisation qui s'y déroule. L'alcoolisation en contexte rituel, même si elle a biologiquement et socialement de similaires effets, admet de sensibles écarts en termes de signification et de positionnement dans l'appareil liturgique. Ce constat amène à une certaine prudence lorsque l'anthropologie s'efforce d'établir des théories générales sur "l'alcool et la religion ».

Mots-clé : boire, rituel, judaïsme, shamanisme, comparaison.

\begin{abstract}
On the basis of ethnographic evidences taken from two very different rituals, in terms of forms, cultural and religion contexts (the Jewish ritual of Pessah observed in Europe and the shamanic guru-puja ritual observed in Nepal), this paper aims at putting emphasis on the salient morphological, functional and symbolic contrasts not between rituals themselves, but between the modes of absorption of alcohol within these rituals. Drinking alcohol is taking place in both religious settings, and has similar effects on the biological and social levels. But it also assumes significant contrasts in terms of meaning and location in the liturgical scheme. This remark calls for prudence, when anthropology is attempting to framing broad theories on "alcohol and religion".
\end{abstract}

Keywords: drinking, ritual, Judaism, shamanism, comparison. 


\section{Le boire, le rite, le rituel du boire : approches et méthodes}

Fondé sur un matériau ethnographique de première main, ainsi que sur l'exploration de corpus sacrés et d'une analyse de la littérature scientifique, cet article vise à interroger les fonctions de l'ivresse alcoolique en contexte religieux. Plutôt que de faire demeurer l'analyse sur le plan relativement circonscrit d'une ethnographie localisée, ce qui est souvent le cas, il sera ici question du boire ritualisé ou en contexte rituel dans une perspective comparative. Ce retour au comparatisme, ambition méthodologique de l'anthropologie à ses débuts, mais finalement peu pratiquée désormais, n'est pas le retour d'un comparatisme à vaste échelle, celui de traits " universels » ou de sociétés entières comparées les unes aux autres. C'est plus précisément à un comparatisme à l'échelle des études de cas que le boire (comme d'autres pratiques sociales et culturelles) se prête. Il permet de revenir à une anthropologie comparative mais enracinée dans des terrains bien circonscrits, qui questionne les propriétés et fonctions de l'alcoolisation en contexte religieux, en dégageant d'une ethnographie menée au plus près de l'activité religieuse les caractéristiques saillantes, les contrastes et similitudes entre les matières et manières du boire.

Les cas présentés dans cet article sont d'autant plus intéressants pour l'analyse que les deux systèmes religieux comparés présentent des caractéristiques particulièrement dissemblables. D'un côté, le judaïsme, qui est un monothéisme né dans le monde sémitique, fondé sur une figure divine transcendante et omnipotente, un corpus religieux textuel et dogmatique qui fait autorité (en tant que " religion du Livre »), inscrit dans une tradition scripturaire particulièrement normative, et adossé à un système d'observances proscriptives (sexuelles, alimentaires, attitudinales, sociales) mais qui comprend également des pratiques festives et prescriptives où l'alcool est proposé voire recommandé. De l'autre, le chamanisme, religion de possession ou de communication médiumnique, tradition orale et « primordiale » pour les historiens inspirés d'Eliade (1955), « culte ethnique » pour des ethnologues d'anciennes générations, dont les mythologies et ritualités sont aussi variées qu'il y a de cultures qui ont fait du chamanisme leur système de croyances principal ou qui l'ont associé à d'autres.

Il est connu des ethnographes que les conduites d'alcoolisation sont enserrées dans des cadres sociaux ritualisés (elles sont rituelles en elles-mêmes) et des contextes ritualistes (elles sont inscrites dans des rituels religieux). Mais ces conduites sont aussi sujettes à des variations de contenu liées au cadre culturel ou religieux dans lequel elles s'inscrivent. Pour le dire plus directement, le lien qui existe entre l'acte de boire et son contexte symbolique admet de significatives variations déterminées par des situations sociales très localisées. Cela n'empêche nullement les sciences sociales de forger des modèles de portée plus large, et d'établir, sur des cas très particuliers, des théories générales ou de vaste portée sur l'alcool et la société ou l'alcool et la religion en général (Nahoum-Grappe 1991; Obadia 2004a).

En retour, de toujours plus nombreuses ethnographies situées portant sur les pratiques de consommation d'alcool en contexte religieux, ou en contexte laïc mobilisant des références religieuses obliques (Castellain 1989 ou Fainzang 1996, pour la France), semblent, peu ou prou, confirmer la pertinence de grands modèles d'analyse, ceux-là même qui stipulent que l'alcool est un facteur de facilitation de la sociabilité en contexte rituel (comme lubrifiant social, cf. Joly 2004), mais que son potentiel de modification 
des états de conscience peut dans le même temps produire des effets psychologiquement et socialement destructeurs pour les individus et par extension pour les communautés.

En filigrane de ce modèle, c'est une autre idée plus générale qui est défendue, celle qui veut que le contexte rituel, ici entendu au sens anthropologique du terme comme embrayeur et ciment de sociabilité (Segalen 1998), représente un garde-fou normatif qui prévient souvent et cadre toujours les dérives d'alcoolisation excessive. Un boire ritualisé serait donc, dans l'acception anthropologique, un boire social relevant d'un ethos culturel, qui aurait pour vocation de prémunir contre les excès d'alcoolisation, désocialisés et désocialisants, responsables d'un pathos qui affecte les sociétés traditionnelles trop rapidement projetées dans une modernité qui en a sapé les fondements et les normes (Nathan 1989).

Face à ces grands modèles, souvent établis à partir d'une ethnographie locale érigée au rang de théorie anthropologique, une ethnologie " comparée " des conduites d'alcoolisation reste à l'évidence encore largement sous-informée - et c'est précisément ce que cet article entend proposer : un cas d'étude singulier, avec l'objectif d'alimenter la réflexion sur les écarts existant entre les modèles d'alcoolisation. Des comparaisons transhistoriques ou transrégionales (en particulier en Europe, Caro et al. 1990), ou l'analyse des interactions entre le " boire » (ses symboles, usages et ses traditions) et son horizon culturel ont déjà été entreprises (en Afrique, Joly 2004.). Le monde asiatique, dont il sera question ici, n'a sans doute pas livré les plus nombreuses ni les plus significatives contributions dans le champ sauf quelques cas bien identifiés d'alcoolisation festive dans les cultes des dieux territoriaux au Japon (Cobbi 1991) ou d'alcoolisation sociale et professionnelle en Corée (Sang-Gil Lee 2007), pour n'en citer que deux parmi bien d'autres.

\section{Par-delà l'approche monothéocentrée}

Parce qu'elles offrent à l'analyse des vues contrastées sur les usages alcooliques, les civilisations monothéistes bénéficient évidemment d'une surface scientifique bien plus visible ; elles occupent une position privilégiée qui est par ailleurs à l'origine de certains paradigmes d'analyse qui ont la vie dure mais qui méritent pourtant d'être déconstruits. C'est à travers l'étude des religions du monde européen, polythéismes antiques et monothéismes tardifs, que s'est en effet installée l'idée que « la religion » en général avait un rôle prophylactique sur les abus de drogue et d'alcool (Obadia 2004a) et que certaines religions jouaient plus particulièrement ce rôle - c'est le cas notamment du judaïsme (Chapuis 1989), mais aussi, pour d'autres raisons, du chamanisme, en particulier sous la forme réinventée et modernisée des néo-chamanismes qui se veut comme une réponse aux " maux de la modernité », et en particulier aux addictions psychotropes et alcooliques (Hamayon 2015 : 58).

Ce modèle, pour intéressant qu'il soit, est toutefois quelque peu réducteur : il pousse à surdéterminer le rôle de la religion dans l'explication des conduites d'alcoolisation ; et si celui-ci est avéré, le modèle laisse alors de côté la complexité et l'ambivalence des relations entre l'alcool, ses usages et ses effets psychosociaux qui peuvent se révéler troubles (Caro 1990 ; Obadia 2004a). Car la religion en général, le judaïsme et le chamanisme en particulier, mais pour des raisons non réductibles les unes aux autres, invitent aussi à la consommation et à l'ivresse, et ont également des effets qui 
présentent une valeur à connotation positive dans le cadre de la religiosité pratique. Dans les deux cas, l'ébriété est un facilitateur d'états psychoaffectifs dont les rituels sont les vecteurs, effusions qui forment le terreau du sentiment religieux, du moins depuis la sociologie durkheimienne (Durkheim 1916). Dans le cadre de cet article et pour ces deux traditions, il s'agit précisément de s'interroger sur ce paradoxe : lorsque l'alcoolisation est requise ou encouragée par le rite, quel est précisément le rôle que la culture locale fait jouer à l'ivresse et comment est-elle socialement contrôlée - et finalement, avec quelle efficacité ? La comparaison des cultures religieuses, qui fondent le background des conduites d'alcoolisation, sert souvent une réflexion d'ordre général sur la tolérance de tel ou tel système religieux à l'endroit de l'alcool et de l'ivresse, et ses implications sur des cultures du boire « abstinentes », " permissives » et « ambivalentes » (Caro 1990). Mais l'ethnographie offre des éclairages un peu décalés par rapport à ces modèles. Elle focalise alternativement sur la transversalité des formes (les manières de boire, souvent ramenées à des invariants par l'analyse) et la singularité des contextes (les environnements du boire, qui sont pour leur part plutôt déclinés dans leur diversité). C'est la raison pour laquelle l'ethnographie, quand elle se réclame du comparatisme, est confrontée à la difficile tâche d'articuler des fonctions aux conduites alcooliques et à des répertoires de significations stables.

Cet article a pour but de montrer, à partir de deux terrains très éloignés sur le plan géographique, religieux et culturel, que si alcoolisation il y a, dans les deux cas, celle-ci assure des fonctions différentes sous la surface des similitudes formelles que l'ethnographie enregistre et dégage de l'analyse. En d'autres termes, dans des rites très différents, on peut tout autant boire, avec des effets bio-organiques attendus similaires (l'ivresse éthylique) mais des modulations d'intensité, de sens et, par extension, de fonction relativement dissemblables.

La démonstration portera ici sur deux rites religieux alcoolisés, précisément retenus en raison des différences très saillantes qui les distinguent. L'un est en effet inscrit dans un contexte monothéiste et européen, l'autre dans un contexte polythéiste et asiatique. Les deux ont en commun d'engager la pratique et de l'associer à des significations religieuses, mais sur la base de fonctions contrastées : il s'agit du rite juif de Pessah, observé en France, au cours duquel se dévoilent les fonctions liturgiques de la consommation de vin, et le rite chamanique de guru-puja, observé dans le nord du Népal, dont le déroulement est émaillé le temps d'un boire récréatif qui assure néanmoins lui aussi des fonctions de facilitation sociale du rite. Les matériaux qui servent à l'analyse du boire chamanique ont été recueillis à l'occasion de plusieurs missions de terrain en milieu sherpa et magar menées entre 1999 et 2003 dans le district du Solukhumbu (nord du Népal), plus précisément dans la région du Pharak, une zone de moyenne montagne située entre 2.800 et $3.200 \mathrm{~m}$ d'altitude, aux pieds de l'Everest. Zone rurale par excellence, elle est connue pour être la région de résidence historique des Sherpas. Quant à l'alcoolisation religieuse en milieu juif, elle a été théorisée à partir d'une vingtaine d'occurrences enregistrées dans des contextes variables - en France et en Israël, en diaspora et dans la territorialité politique et religieuse, dans des milieux ashkénazes et séfarades, en petit groupe (de quatre à cinq personnes) ou en plus large comité (de plusieurs dizaines de convives). Le rituel a été ici recomposé en ses éléments de base nécessaires à l'analyse, et ce sont donc ici les traits morphologiques communs et non pas les variations internes au rituel qui seront dégagés. 


\section{Boire en contexte juif : «s'accouder et boire » à Pessah}

Le premier rituel ici à l'étude est presque un « classique » des sciences religieuses, tant il marque, par sa charge symbolique et par son organisation en une série d'actes codifiés, un rituel très important des cultures juives, qu'elles soient européennes (ashkénazes), nord-africaines (séfarades) ou autres. Il s'agit de Pessah, la Pâque juive, qui est, selon la hiérarchie, établie par le judaïsme rabbinique, l'un des rituels de premier plan à préférer à d'autres, plus mineurs. Il est d'ailleurs l'un des plus observés dans les communautés juives d'Europe. Comme pour d'autres grands rites festifs qui fondent le complexe liturgique du judaïsme, Pessah est l'un des principaux rituels de " solennité » qui marquent les grands moments du calendrier religieux du judaïsme, toutes branches et traditions confondues. Festivité printanière qui rejoue l'un des épisodes majeurs de l'histoire juive, la sortie d'Egypte après une captivité en exil, le rite de Pessah est, sur le plan fonctionnel, un temps de réjouissances malgré le (ou plutôt en raison du) caractère dramatique du récit sur lequel il se fonde : la douleur de la captivité d'un peuple réduit en esclavage et la violence de la libération du joug politique de l'Egypte pharaonique. Ce caractère festif - la recherche de la joie, sim'ha, dans le rite et par le rite - est largement répandu dans la liturgique juive, à défaut d'en figurer un trait général.

Comme dans le cas de Pourim, l'un des autres grands rituels de solennité, il s'agit de festivités domestiques et commensales. Mais Pourim est marqué par un caractère quasi obligatoire de participer au « festin », michté, ce qui signifie boire à outrance, jusqu'à être affecté d'une légère ivresse (bassomé). Cette obligation à l'ivresse relève en fait d'une leçon de morale : celle de ne pas privilégier les sources d'un bien-être important mais néanmoins superflu (la vigne) sur celles qui assurent la subsistance de base (le blé). Pessah fait aussi partie de ces « solennités » rituelles qui regroupent les membres de familles (élargies) au sein d'espaces domestiques autour d'une table, et qui enjoignent à la commensalité et, jusqu'à un certain point, à l'ivresse, mais avec des finalités et effets un peu différents de Pourim, qui est à ce titre un cas particulier, souvent point de départ des réflexions autour des rapports entre judaïsme et alcool.

La culture religieuse juive est en effet une culture sémitique qui a établi un grand nombre de ses croyances et rituels en accord avec la géologie, le climat et l'écologie particuliers du bassin méditerranéen : elle est baignée d'une culture de la vigne (gefen) et du vin (yayin) qui est l'une des « bénédictions » de la Terre, symbole d'abondance distribuée avec prodigalité lors des fêtes (hagim). Les rituels religieux comptent ainsi souvent cet alcool dans les liturgies, au point qu'il a même été écrit que, dans le judaïsme, « il n'est pas de réjouissance importante qui n'implique la consommation de vin » (Attias et Benbassa 1997 : 293). Pour autant, et parce qu'il est source d'ivresse et donc perte de contrôle des normes culturelles acquises, le vin est une figure ambivalente, qui, d'un côté, réjouit Dieu et « le cœur de l'Homme » (Psaumes 104 : 15), mais d'un autre, est aussi capable de détourner des règles de la bienséance. Il participe d'un partage social et d'une effusion émotionnelle propices à l'émotion religieuse. Mais d'un autre côté, c'est ce même vin qui, consommé en excès, fait que des personnages de l'Ancien Testament perdent de leur exemplarité et se livrent à des actes moralement répréhensibles. C'est le cas notamment de Noé, dont l'ivresse l'amène à dévoiler sa nudité à ses enfants (et peut-être même à avoir des relations sexuelles avec eux, affirment certains rabbins), ce 
qui est contraire à la règle de pureté ${ }^{\text {. }}$

A ceci s'ajoute le rythme hebdomadaire du shabbat (jour chômé) qui s'adosse et se cumule aux autres fêtes, dont Pessah. Cette fête comprend aussi deux jours chômés : ceux de la festivité commensale proprement dite (seder), sur les sept que compte la fête. On y boit donc doublement car le seder du shabbat comprend un qiddouch (célébration du vin), qui s'ajoute à la quantité à consommer au seder de Pessah. Certes, le volume absorbé en cette occasion y est nettement inférieur et peut se limiter à quelques gouttes, mais lorsque le verre circule, accompagné d'une prière, les plus jeunes membres de la famille jouent à tremper leurs lèvres plus qu'attendu dans la coupe remplie qui passe de main en main. C'est alors le contrôle des aînés qui freine leur ardeur à la recherche de l'ivresse, si modérée soit-elle.

Le rituel de Pessah (ou «Pâque juive ») se déroule ainsi sous la forme de deux soirs de festivités (seder) lorsque la communauté est en diaspora (un soir seulement pour les Juifs résidant en Israël) autour d'un repas ; c'est une liturgie adossée à une commensalité, et, à la différence d'autres grands rites du judaïsme, l'office des prières et célébrations ne se conduit pas au temple (synagogue) mais dans l'espace domestique. Le rituel débute par une chasse (symbolique) aux derniers résidus de nourritures levées (hametz) sous la direction du père de famille du foyer mais avec une participation active des enfants. Une fois ce préalable satisfait, rituel avant le rituel, le seder peut commencer autour de la lecture de la Haggadah, le livre qui comprend à la fois la narration et la liturgie de la célébration. A plusieurs reprises, au cours de cette longue soirée festive « on verse le vin » dans des verres mais l'absorption se limite à « quatre coupes » qui sont bues « en s'accoudant » et doivent être presque entièrement vidées car doit être absorbé à cette occasion un moins un revi'it (une unité de mesure liturgique propre à ce rite). A l'observation directe, celle de ces « petits détails » qui fondent toute la complexité des grands rituels (Piette 1999), c'est une autre mise en scène qui se joue : celle, ludique, du jeu social de l'observance rituelle sans excès, de la consommation sans ivresse.

Dans le grand cycle annuel de la ritualité juive, le rite de Pessah occupe une place particulière, car ses significations officielles, scripturaires, sont d'ordre mémoriel : le rituel est nocturne, il ouvre une semaine de diète particulière en souvenir de privations passées, et il figure un événement clef de l'histoire mythique des anciens Hébreux, qui travaillent encore actuellement une mémoire collective qui se réécrit au gré des traumatismes que les siècles infligent à ceux qui furent les Hébreux en exil. Le judaïsme est une religion tributaire de son ancrage premier dans une économie agricole, qui a initialement marqué (et qui continue de marquer profondément) de son empreinte sa ritualité et sa liturgie, qui compte quantité de références à une économie productive de type agropastoral.

1 Sans compter dans cette brève énumération que le vin est aussi selon l'exégèse de l'Ancien Testament un révélateur mystique, puisque le traité Erouvin de la tradition orale du Talmud Thora, le texte de référence en matière de décryptage du Pentateuque (les cinq livres de base de la tradition abrahamique), établit un lien numérologique et donc ésotérique entre le vin (yahin) et le secret (yod) au principe qu'un être se fait notamment connaitre par « sa coupe » ou ce qu'il boit (voir l'interprétation d'Erouvin 65 par Pauline Bebe, 2015). 
Mais quand le regard de l'anthropologue quitte le plan textuel (i.e. ce que le rite veut dire sur le plan des normes textuelles) et se focalise sur le rite « en train de se faire " ou dans le cadre de la pratique au ras des acteurs, alors la signification des actes qui le constituent, le sens qu'il assume et les effets qu'il engendre changent : la fonction symbolique de la fertilité, motif premier de l'alcoolisation religieuse, se trouve associée à des fonctions socialisatrices par et pour le rite. Parce qu'il y a autorisation et même " obligation » de boire, même pour les femmes et les enfants ayant atteint leur maturité spirituelle, le rite est source d'une potentielle ivresse qui facilite l'effusion psychoaffective. Il participe au maintien de la sociabilité émotionnelle nécessaire à la perpétuation du lien social et de la mémoire collective, d'autant plus important pour une religion très minoritaire dans le monde, largement dispersée par ailleurs et menacée d'assimilation par l'intégration dans ses sociétés d'accueil en diaspora (Blau 1964). Ainsi le rituel de Pessah, comme d'autres rites ou solennités du judaïsme, comprend-il des modalités d'alcoolisation centrales et codifiées, officielles et chargées de symboles, héritées du temps où les Hébreux avaient pour environnement écologique et pour économie principale un monde agricole et une civilisation de la vigne.

\section{Célébrer le rapport aux esprits : le «guru-puja » au Népal}

Quittons maintenant le monde juif européen pour rapatrier l'analyse en Haute-Asie. C'est un environnement naturel et culturel radicalement différent qui figure l'arrièreplan matériel, social et symbolique du rituel chamanique qui servira de base à la comparaison ici mise en œuvre. Il s'agit du guru-puja, traduit par « l'offrande » ou « l'adoration », plus généralement la « cérémonie » ou le « culte » rendu au « maître ». C'est une pratique de premier ordre dans le sous-continent indien, qui se déploie autant dans le monde hindouiste que dans les environnements chamaniques de l'Inde et du Népal, et qui s'inscrit dans de complexes cycles rituels locaux (Toffin 1982 ; Bouillier 1982).

Le terme puja a aussi pu être assimilé à celui de « rituel» en général (Peeters 1981). La variante spécifique du rituel guru puja dont il est ici question est une pratique qui s'inscrit dans le complexe calendrier des festivités des chamanes de type dhami-jhankri qui officient dans la zone himalayenne et auxquels échoient des rôles de prêtrise, de thérapie et de divination (Miller 1997). Dans les groupes bothia, populations d'origine tibétaine, comme les Sherpas et les Magars, dominants sur le plan démographique et culturel dans cette région du nord du Népal, les rituels chamaniques ont pour théâtre les espaces domestiques et le chamanisme n'a pas de temple ni de prêtre ; il n'a que des médiums, désignés par les forces de la surnature, et les résidences des chamanes eux-mêmes, qui se transforment pour l'occasion en salles de rituel. Le chamanisme des Magars du Népal, culte himalayen de la transe (de Sales 1991) est fortement hindouisé (Lecomte-Tilouine 1993) mais le chamanisme en général est largement répandu dans l'ensemble du Népal (Miller 1997) où il revêt des formes différenciées selon les régions et les groupes ethniques (Hitchcock et Jones 1976).

Les Sherpas, bouddhistes de tradition tibétaine résidant dans le Nord, ont développé une culture singulière avec des spécificités liturgiques. Leur bouddhisme a largement absorbé le chamanisme, qu'il soit tribal et local (von Fürer-Haimendorf 1964) ou d'ascendance tibétaine (Paul 1979), et est organisé autour de complexes cycles rituels 
qui sont d'ailleurs principalement des exorcismes (Ortner 1978). Bouddhisme et chamanisme se combinent ainsi dans un même univers culturel mais en occupant des temps et des lieux différents : au bouddhisme reviennent le temps diurne et les festivités publiques et fortement organisées, et au chamanisme le temps nocturne et des ritualités plus confidentielles - l'une et l'autre traditions figurant une facette particulière d'une société complexe (von Fürer-Haimendorf 1964 ; Kunwar 1989). Le bouddhisme, une religion qui impose peu de contraintes sur le plan domestique pour les laïcs, laisse largement s'exprimer le goût des villageois pour les boissons alcoolisées dans le cadre de la sociabilité ordinaire. Les normes de l'hospitalité villageoise et clanique, dans ces sociétés à forte solidarité fonctionnelle, incluent des temps de festivité et de commensalité qui engagent la distribution de boissons alcoolisées. Cette alcoolisation se manifeste à l'occasion de rituels sociaux, en particulier les nombreux protocoles de l'hospitalité tels qu'ils caractérisent les formes culturelles ritualisées et locales (Ortner 1978). Ils ne sont pour autant pas nécessairement au centre des pratiques religieuses collectives (Kunwar 1989).

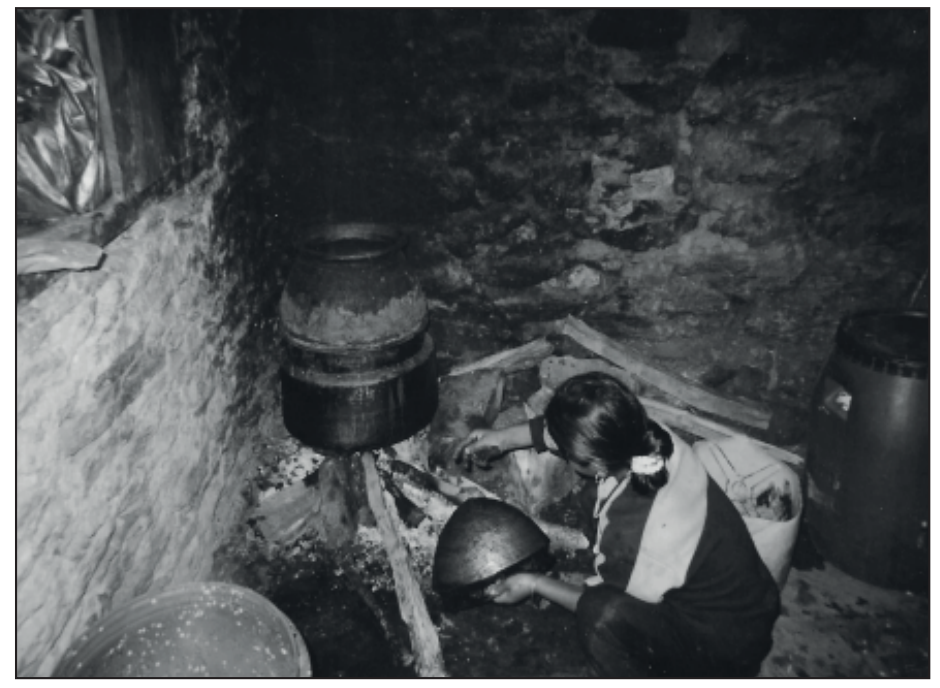

Figure 1 : Une jeune femme sherpa prépare un roksi (whisky) dans un alambic, région du Solukhumbu, Népal - C L Lionel Obadia

Le chamanisme, en contraste d'un bouddhisme aussi socialement structurant, figure la part sombre, extatique, effervescente et violente de ces sociétés de moyenne montagne (Obadia 2010). Dans le contexte de la pratique chamanique, le boire apparaît alors comme un lubrifiant social particulièrement important puisqu'il est un ingrédient fondamental « dans le » rituel mais pas nécessairement « du » rituel.

\section{L'alcoolisation dans le « guru-puja »}

L'alcool est aussi un ingrédient des rituels pratiqués par les chamanes dhamijhankri du groupe ethnique magar, qui pratique un chamanisme hindouisé, et chez les chamanes lhawa du groupe ethnique tibéto-sherpa, dont le bouddhisme prend des couleurs médiumniques et extatiques, en marge de l'ascétisme monastique qui est méditatif et exclut presque totalement l'usage d'alcool. Les deux constituent des modèles de référence, car les pratiques d'alcoolisation sont empiriquement saillantes et donc faciles à saisir pour l'analyse. Dans d'identiques cadres écologiques, mais à background culturel et religieux différent, les Magars et les Sherpas ont développé des 
formes de chamanisme qui s'inscrivent toutes deux pleinement dans une économie agricole et commerciale, fondée sur une production céréalière (Cox 1985). De cette dernière dérive une production d'alcools artisanaux, une bière de millet (chhang) et le rakshi (ou rokshi) un spiritueux façonné à partir de la distillation du moult de la bière non consommée, et, de manière plus spécifique aux bouddhistes sherpas, une macération absorbée chaude qui se nomme thongba. A l'image du vin dans la Pâque juive, la consommation de ces alcools est un élément clef des rituels chamaniques. Mais là où le rituel de Pessah enjoint paradoxalement à l'ivresse tout en suscitant le contrôle de l'ébriété par la communauté, les rituels chamaniques comme le guru-puja, mais aussi le mela-chinta, une festivité à laquelle participent les chamanes, entraînent de manière non contingente des débordements individuels et des violences collectives entre les sous-groupes constitutifs de ces sociétés de montagne où pourtant la solidarité est de mise dans une économie productive et d'échange qui a besoin de la coopération entre ses acteurs (Obadia 2004b).

Les rituels d'extase chamanique, dans cette région de l'Himalaya, n'ont pas besoin de l'aide de psychotropes pour faire accéder à l'état de transe qui s'obtient par le rythme des tambours : ni psychotropes ni alcool ne sont requis pour susciter l'extase des médiums. Pourtant l'alcoolisation est bien présente dans ce cadre religieux. Car l'alcool est un ingrédient mineur mais néanmoins toujours présent dans les petits comme dans les grands rituels de la région, au premier rang desquels se range le guru-puja. Il semble assurer des fonctions essentiellement socialisatrices, en l'absence de références directes à des fonctions symboliques dans la tradition orale (qui réfère occasionnellement seulement aux pratiques de boisson), ou de prescriptions liturgiques aussi explicites que celles que l'on trouve dans le judaïsme.

Le rite du guru-puja se déroule dans la maison d'un(e) chamane réputé(e) dans la région qui, à l'occasion, réunit une vingtaine de membres de son groupe ethnique, incluant vieux, femmes et enfants en bas âge. Il se déroule de nuit, sous la forme d'une longue cérémonie qui transforme temporairement l'espace résidentiel des chamanes en espace de prêtrise. Pour ce faire, l'ergonomie du lieu est aménagée pour servir de théâtre à cette invitation des esprits surnaturels ou « maîtres » (guru) à être célébrés par des offrandes (puja). La cérémonie débute par la confection d'un autel vertical (than) sous la forme d'un treillis de bois sur lequel sont accrochés des fleurs, fruits et légumes qui feront office d'offrandes. Cette première étape requiert le silence et est essentiellement assurée par la/le chamane qui se vêtira alors de ses atours liturgiques - collier de perles (rudraksa), ceintures de clochettes et trident (trisul) - et s'emparera ensuite de cymbales et du tambour chamanique (dhyangrö) qui sera martelé avec plus ou moins de force et de régularité en fonction de la séquence du rituel, qui dure une grande partie de la nuit. Plus que la description des rituels chamaniques eux-mêmes, que bien d'autres ont déjà décrits en détails, on s'attardera ici à la consommation d'alcool, à ses temps, à ses modalités et à ses effets.

Le principal breuvage alcoolisé distribué au cours du guru-puja est le chhang, bière traditionnelle qui est brassée à la fin des récoltes dans quasiment chaque foyer qui dispose d'un lopin de terre ; les membres qui exercent des métiers non agricoles se fournissent auprès d'autres villageois. Fruit de la fermentation du millet, elle est fabriquée par pressage à la main et conservée dans de grands bacs. Elle est en général servie dans le 
cadre de la sociabilité villageoise, lorsqu'un invité (voisin, parent, membre du même clan, collaborateur, chef, etc.) est reçu dans un foyer : la qualité, le volume de la boisson servie, ainsi que les manières de la servir, sont des révélateurs des rangs sociaux.

La bière est distribuée de manière collective et bien moins solennelle lors du rituel du guru-puja. Un grand récipient en plastique bleu, de type réservoir d'eau, ou une barrique en bois contient un grand volume (plusieurs dizaines de litres) de chhang, que l'on sert généreusement dans de simples verres, en laissant quelques gouttes tomber au sol (en signe de fertilité et en offrande aux esprits chtoniens). La bière est distribuée sans plus de protocole et surtout, absorbée sans autre forme de procès que le penchant plus ou moins marqué des villageois à l'endroit de ce breuvage, et surtout à la manière locale : aspirée à grand bruit, ce qui est un signe d'appréciation du contenu.

Par contraste avec le rite précédent, l'alcoolisation, dans ce contexte rituel (liturgique), n'est pas ritualisée (organisée sur une gestuelle codifiée) et c'est essentiellement sa temporalité qui fait sens et qui explique ses fonctions attendues. La bière est en effet consommée par les chamanes et par l'audience dans des moments très particuliers, les pauses entre deux temps où le rite atteint des pics d'intensité. Il s'agit en l'occurrence, des temps de possession stricto sensu, lorsque le corps du chamane dhami-jhankri est saisi de spasmes et qu'à travers sa voix s'expriment les esprits de « l'autre monde ». L'audience est toute entière absorbée par ce point culminant du rituel, sur le plan émotionnel. Elle est à l'écoute et concentrée sur la dramaturgie qui se joue ici : l'incarnation des forces invisibles dans le visible, la mise en présence du divin et du sacré dans la spatialité humaine, ce qui est la base d'une religiosité populaire fondée sur la possession, telle qu'on la retrouve dans l'ensemble du sous-continent indien (Assayag et Tarabout 1999).

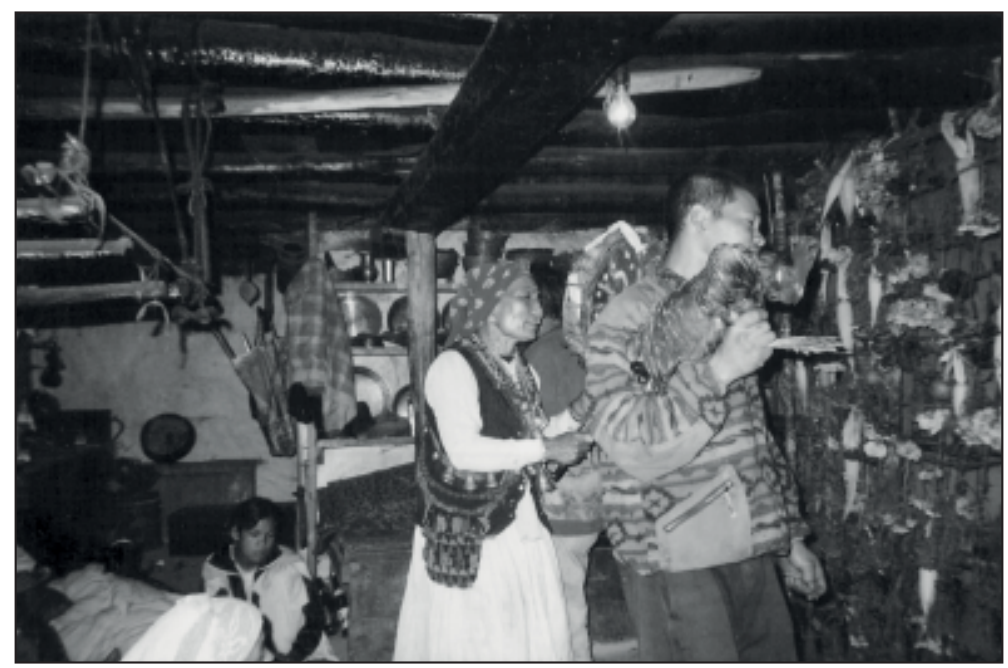

Figure 2 : Une chamane dhami-jhankri et ses assistants dole à l'occasion d'un rituel mela-chinta, région du Solukhumbu, Népal - (C) Lionel Obadia

Car le rite dure de nombreuses heures, et même aidés par des forces surnaturelles, les chamanes n'ont pas la force physique ni morale de maintenir cet état d'extase au-delà de quelques dizaines de minutes, de même que le public ne peut maintenir une attention soutenue et être continuellement absorbé par la transe des chamanes. Le rituel de gurupuja prévoit donc des aménagements suspensifs du déroulement rituel proprement dit, mais qui font demeurer les médiums et l'audience dans un cadre religieux, même si 
lorsqu'on boit, on converse, on s'amuse les uns des autres, on allège la pression du rituel. La bière de millet est en effet servie dans ces pauses qui séparent deux séquences rituelles d'une forte intensité, après de longues séances de danses et chants, et des moments fugaces, mais particulièrement intenses où les corps sont secoués par la présence des esprits et où le son des tambours et cymbales fait monter la tension à son paroxysme. Ensuite, le ou la chamane jhankri et ses « aides » (dole) suspendent l'activité rituelle durant quelques minutes où tous les sons sont neutralisés et tous les corps sont de nouveau domestiqués par les humains : on ne chante plus, on ne danse plus, on ne joue plus d'instruments et le/la chamane retrouve sa voix originale, qui s'exprime en dialogue avec d'autres humains, avec courtoisie et badinerie.

A la différence du rite de la Pâque juive, où l'alcool peut susciter des amusements hors cadre rituel, mais n'a pas été pensé pour ça, l'usage du chhang a une fonction principalement récréative pour le chamane et pour l'audience. On boit beaucoup lors du guru-puja. Ce n'est pas tant que les effets de l'alcool aident à la communication spirituelle ou à l'incarnation des esprits dans le monde : ils participent aussi et surtout d'une convivialité festive inhérente à l'activité rituelle, et qui se déploie dans ses temps « faibles » ou du moins, dans des temps interstitiels entre deux moments d'intensité rituelle. Dans ce sens, l'alcool n'est pas ici un psychotrope dont l'usage aurait des fonctions « participatives » (pratiques et existentielles) au sens que Lévy-Bruhl donne à ce terme (Lévy-Bruhl 1949), mais un élément additif sur le plan de la sociabilité : le boire dans le rite chamanique himalayen ne façonne pas de la socialité uniquement par effervescence, comme le veut le modèle d'analyse durkheimien (Durkheim 1916) ; elle se construit sur la base d'actions apparemment plus " mineures » généralement passées sous silence dans les études ritologiques (Piette 1999). Ainsi le déroulement du rite déploie-t-il des séquences formelles qui s'enchainent les unes à la suite des autres et qui sont rythmées par moments interstitiels, des temps plus faibles de l'intensité émotionnelle et physique tant pour les officiants que pour l'audience. La consommation d'alcool dans ces hautes vallées de l'Himalaya s'inscrit dans des cadres culturels qui débordent ceux où le breuvage joue un rôle très important : on boit pour sceller des contrats, renforcer des relations interpersonnelles ou familiales, mais aussi (et « en plus ॥, pourrait-on dire) au cours des rites religieux des communautés villageoises. L'alcool conforte ainsi des formes de sociabilité traditionnelles qui s'expriment à travers des expressions culturelles, lesquelles s'invitent dans l'activité religieuse : dans la mesure où l'alcoolisation est social(isée) et culturelle(ment légitime et importante), il est donc peu surprenant qu'elle s'invite dans l'activité religieuse, qui représente un répertoire singulier de ces deux dimensions.

C'est l'une des raisons pour lesquelles le mouvement maoïste, qui s'était engagé en 1996 dans une sanglante conquête du pouvoir (jusqu'à son accession à la tête du pays en 2006), avait fait de l'interdiction de l'alcool, notamment dans les villages, l'une de ses priorités. Cela s'est traduit dans les faits par une interdiction adressée aux paysans et villageois de consommer de l'alcool, pour autant que la présence des forces maoïstes pouvait en contrôler la production et la consommation (Obadia 2004b). Mais les rituels du boire se sont maintenus dans les campagnes, en vertu du lien étroit qui relie l'alcool au système économique (agriculture) et aux systèmes symboliques (religions) dans cette région du monde. Pour ces populations habituées à des offrandes de boissons et libations aux dieux et aux ancêtres, l'interdiction signifiait une attaque frontale contre 
la religion, menée par les maoïstes au principe d'une prévention sanitaire, qui masque en fait un véritable projet de transformation des bases civilisationnelles (culturelles et matérielles) des campagnes népalaises (von Stockhausen et Wettstein 2013 : 116 sq.)

\section{Conclusion}

L'approche comparative à laquelle on s'est essayé dans cet article dégage ainsi $\mathrm{du}$ matériau ethnographique des formes et des fonctions du « boire »relativement contrastées, qu'une théorie par trop englobante aurait eu tendance à rapprocher, voire à confondre. Ce n'est pas parce que le boire s'inscrit dans un contexte religieux qu'il est ritualisé selon les mêmes modalités. Et sous la catégorie d'alcool rituel, ce sont des variantes significatives qui se donnent à voir dans les manières et raisons de boire, entre d'un côté une alcoolisation proprement rituelle, où l'ivresse a des fonctions manifestes pour le rituel, comme celle de susciter l'émotion et de participer à son intensité dramaturgique ; de l'autre, une alcoolisation récréative qui se situe dans le rituel mais pas nécessairement (ou pas directement) pour le rituel, où l'ivresse a une fonction dérivée, celle de lubrifiant social et symbolique, favorisant la participation au rituel malgré l'intensité de celui-ci.

Le choix méthodologique ici retenu, qui rapproche des terrains que pourtant tout semble opposer, semble à première vue risqué - un mariage de la carpe et du lapin à la sauce religieuse, en quelque sorte. Ce comparatisme de cas ethnographiques contextualisés (et non pas de sociétés ou de cultures entières) apparaît pourtant d'une indéniable qualité heuristique tant il apporte à l'analyse les arguments pour contrecarrer toute velléité à généraliser trop hâtivement les rapports entre l'alcoolisation (traditionnelle) et la religion. Il fallait pour autant conserver à la pratique du boire alcoolisé en contexte religieux sa singularité sans trop la réduire à des aspects localisés incommensurables.

Sur le plan ethnographique, l'alcoolisation en contexte religieux et ritualisée à cette fin apparait alors comme un trait commun de ces deux festivités, dont les environnements géographiques et culturels sont pourtant aussi éloignés que peuvent l'être le monde sémitique et monothéiste, héritier d'une civilisation du vin, synonymes de prodigalité, et le monde himalayen, bouddhiste et chamanique, avec ses systèmes agropastoraux à dominante céréalière, où ce sont cette fois la bière et ses dérivés spiritueux qui servent de drogues socialement acceptées et - jusqu'à un certain point seulement - légitimes. Dans les deux cas, l'alcoolisation est une composante du rituel, mais elle participe à son symbolisme et surtout à sa praxis de manière différenciée. Pour y revenir encore une fois et au risque de la répétition, elle est, d'un côté, codifiée par les textes et associée à une ivresse religieuse dans le judaïsme, donc une ivresse pour et par le rite, pour lequel elle est centrale, mais elle apparaît d'un autre côté comme une pratique inscrite dans le rite et qui en facilite le déroulement, tout à s'avérant nettement plus marginale, mais en aucun cas insignifiante. La distinction est donc formelle, et elle se situe au-delà (ou en deçà) de l'ensemble des différences qu'il est facile de lister par le jeu des contrastes et similitudes entre le judaïsme et le chamanisme, non pas au niveau des faits mais des structures. Ce constat suppose de s'interroger sur la pertinence de théories générales parfois un peu rapidement établies à propos des rapports entre alcool et religion, et des prétendues fonctions extatiques de l'alcool pour le rite via l'ivresse et des supposées fonctions prophylactiques de la religion sur l'alcoolisme. Ainsi, l'ivresse peut-elle se manifester en contexte rituel dans les limites d'une ébriété licite et normative ou les déborder sous 
des manifestations plus excessives. L'alcoolisation religieuse, apparemment identique dans ses formes (indépendamment de la singularité de leur arrière-plan culturel) peut ainsi révéler des logiques différentes qui amènent à s'interroger sur le statut de l'ivresse dans le rituel, qui peut, en outre, s'avérer contingente dans un cas, nécessaire dans l'autre. Dans ce sens - et cet article a précisément tenté de montrer, à travers un essai de comparatisme qui, certes, se fait ici au péril d'une décontextualisation excessive que les liens entre rituel, usage de l'alcool et signification associée à cet usage sont loin d'être mécaniques et nécessitent des éclairages locaux qui en dévoilent la complexité.

\section{Références citées}

Assayag, Jackie et Gilles Tarabout (éds), 1999. La possession en Asie du Sud : parole, corps, territoire. Paris : EHESS Editions.

Attias, Jean-Christophe et Esther Benbassa, 1997. Dictionnaire de civilisation juive. Paris : Larousse.

Bebe, Pauline, 2015. Saisir le merveilleux dans l'instant. Paris : Editions le Passeur.

BLaU, Joseph, 1964. Modern Varieties of Judaism. New York: Columbia University Press.

Bouillier, Véronique, 1982. « Si les femmes faisaient la fête ... A propos des fêtes féminines dans les hautes castes indo-népalaises », L'Homme, 22 (3), pp. 91-118.

CARO, Guy (dir), 1990. De l'alcoolisme au bien boire. Paris : L'Harmattan.

Castellain, Jean-Pierre, 1989. Manières de vivre, manières de boire. Alcool et sociabilité sur le port. Paris : Imago.

CHAPUIS, Robert, 1989. L'alcool, un mode d'adaptation sociale? Paris : L'Harmattan.

CoBBI, Jane, 1991. « Dieux buveurs et ancêtres gourmands », L'Homme, 31 (118), pp. 111-123.

Cox, Thomas, 1985. "Herding and Socio-Economic Change among Khumbu Sherpas", Kailash, 12 (1-2), pp. 63-79.

DE SALES, Anne, 1991. Je suis né de vos jeux de tambours. La religion chamanique des Magar du nord. Nanterre : Société d'Ethnologie.

Durkheim, Emile, 1916. Les formes élémentaires de la vie religieuse. Paris : Presses Universitaires de France.

FAINZANG, Sylvie, 1996. Ethnologie des anciens alcooliques. La liberté ou la mort. Paris : Presses Universitaires de France.

HAMAYON, Roberte, 2015. Le chamanisme : histoire, fondements et pratiques d'une religion millénaire. Paris : Eyrolles.

HitchCock John T. et Rex Jones (éds), 1976. Spirit Possession in the Nepal Himalayas. New Delhi: Vikas Publishing House Ltd.

JoLY, Eric, 2004. Boire avec esprit. Bière de mil et société dogon. Nanterre : Société d'Ethnologie.

Kunwar, Ramesh Raj, 1989. Fire of Himal. An Anthropological Study of the Sherpas of Nepal Himalayan Region. Jaipur \& New Delhi : Nirala Publications

Lecomte-Tilouine, Marie, 1993. Les dieux du pouvoir. Les Magar et l'hindouisme au Népal central. Paris : CNRS Editions.

LÉvY-BruHL, Lucien, 1998 [1949]. Carnets. Paris : Presses Universitaires de France.

Miller, Casper, 1997. Faith-Healers in the Himalayas. Delhi: Book Faith India.

Nahoum-Grappe, Véronique, 1991. La Culture de l'ivresse : essai de phénoménologie. Paris : Quai Voltaire. 
NATHAn, Tobie, 1989. « Bacchus est alcoolique et le grand Pan est mort », préface, in Claude LE VOTIfrah, Marie Mathelin, Véronique Nahoum-Grappe (éds), De l'ivresse à l'alcoolisme. Etudes ethnopsychanalytiques, pp. 9-19 Paris : Dunod / Bordas.

OBADIA, Lionel, 2004a. « Le 'boire' : une anthropologie en quête d'objet, un objet en quête d'anthropologie », Socio-anthropologie, 15, pp. 3-30.

Obadia, Lionel, 2004b, «'No King', 'No drink', 'Power to the People'. Rébellion maoïste et conduites d'alcoolisation dans le Népal contemporain », Socio-anthropologie, 15, pp. 99-114.

OBADIA, Lionel, 2010. «Festivités religieuses, publiques et discrètes. Mondialisation, tourisme, et mimesis chez les Sherpas du Nord Népal », Anthropologie et sociétés, 34 (2), pp. 177-197.

ORTNER, Sherry, 1978. Sherpas through their Rituals. Cambridge, Londres, New York, Melbourne: Cambridge University Press

PAul, Robert, 1979. "Dumje: Paradox and Resolution in Sherpa Ritual Symbolism”, American Ethnologist, 6 (2), pp. 274-304.

Peeters, Larry, 1981. Tamang Shamans. An Ethnopsychiatric Study of Ecstasy and Healing in Nepal. Malibu: Undena Publications.

PietTe, Albert, 1999. La religion de près. L'activité religieuse en train de se faire. Paris : Métailié.

SANG-GIL, Lee, 2007. « Techniques du corps et communication ritualisée. A propos de la culture du boire en Corée », Sociétés, 1 (95), pp. 29-139.

SEgalen, Martine, 1998. Rites et rituels contemporains. Paris : Nathan.

ToFfin, Gérard 1982. «Analyse structurale d'une fête communale néwar : le deś jātrā de Panauti », L'Homme, 22 (3), pp. 91-118.

VON Fürer-HAIMENDORF, Christoph, 1964. The Sherpas of Nepal. Buddhist Highlanders. Londres: Butler \& Tanner Ltd.

von Stockhausen, Alban et Marion Wettstein 2013. "Contesting Power, Negociating Influence : Rai Shamans and New Religious Movements in Eastern Nepal”, in Diana Riboli et Davide TorRi (eds), Shamanism and Violence: Power, Repression and Suffering in Indigenous Religious Conflict, pp. 103118. Londres \& New York: Routledge. 